\title{
Adjustable Transmission Power in Wireless Ad Hoc Networks with Smart Antennas ${ }^{*}$
}

\author{
Fei Huang, Victor O.K. Li, and Ka-Cheong Leung \\ Department of Electrical and Electronic Engineering \\ The University of Hong Kong \\ Pokfulam Road, Hong Kong, China
}

\begin{abstract}
In this paper, we present a model to analyze the performance of wireless ad hoc networks with smart antennas, i.e. directional antennas with adjustable transmission power. Our results show that smart antennas can improve the network performance by mitigating the effects of interference. We illustrate our model with the NFP (Nearest with Forward Progress) transmission strategy. Our analytical and simulation results show that, for ad hoc networks with smart antennas, NFP yields good throughput and remains stable as the node density varies.
\end{abstract}

\section{INTRODUCTION}

It has been shown that the adoption of smart antennas in wireless ad hoc networks can significantly improve the network performance, since it largely reduces the radio interference and exploits spatial diversity to expand the capacity of the network.

There are two different modes of operation for a node installed with a smart antenna. They are the omni-directional mode (OMNI-mode) and beam-forming mode (BF-mode). In OMNI-mode, the antenna sends and receives signals over all directions. In BF-mode, the antenna sends and receives signals through a beam pattern. The interference is mainly determined by the main lobe (and perhaps some of the side lobes) of the beam. In our work, the term "smart antenna" refers to Adaptive Antenna Array (AAA) with steerable beam and adjustable transmission power. The antenna weights of AAA can be dynamically adjusted so that the beam pattern is optimized for the reception of the desired signal and nulling of interference [1].

Optimizing the transmission radius with power control can further improve the performance in wireless ad hoc networks [2]. Power control saves precious battery energy of portable devices and extends the network lifetime [9]. In Nearest with Forward Progress (NFP), a transmission strategy originally proposed for omni-directional antennas, a transmitter will pick its nearest neighbor in the forward semicircle (see Section II. D) as a receiver and the transmission radius is adjusted so that the signal strength is just strong enough for the receiver to get the signal. By doing so, potential conflicts are minimized in the network. NFP yields good throughput in a high terminal density environment. It is best suited for applications in road traffic information systems such as cooperative driving by data exchange between neighboring vehicles, since high throughput is most important to achieve real time and reliable data flow [7].

The transmission range control analysis for omni-directional antennas has been presented in [3]. The analysis has been extended in [10] for directional antennas, but it only considers

\footnotetext{
* This research is supported in part by the Research Grants Council of Hong Kong, under Grant No. HKU 7152/05E.
}

Most Forward with Fixed Radius (MFR) which does not support adjustable transmission power. The results in [10] show that directional antennas increase the network performance dramatically. Furthermore, the transmission range is assumed to be equal to the interference range in [3], [10]. However, considering the signal propagation, some nodes may still interfere with the receiver even if these nodes are out of the transmission ranges of both transmitter and receiver [8]. In our work, we assume that the interference range is larger than the transmission range.

In this paper, we propose an analytical model to analyze the network performance where all ad hoc nodes are installed with power-adjustable directional antennas. NFP is chosen as the transmission strategy for the illustration of our idea. Our results show that power control and directional antennas can improve the network performance by mitigating the effects of interference and reducing power consumption. By using power-adjustable directional antennas, NFP outperforms the corresponding one with omni-directional antennas and offers stable one-hop throughput and forward progress.

The rest of the paper is organized as follows. In Section II, we introduce the network model for our analysis. In Section III, the probability of interference for NFP is derived. The performance analysis is presented in Section IV. Finally, in Section V, we conclude and discuss some possible extensions of our work.

\section{NETWORK MODEL}

Our analytical model is based on the premise that each node knows the locations of all other nodes in the network. The simplest way to find the exact location of a node is to use Global Positioning System (GPS) [4]. The origin will pick its neighbor as a relay according to NFP and transmit a packet with the identity (ID) of the relay and the ID of the final destination in the packet header. A neighboring node receiving this packet will process the packet only if it is identified as a relay. All other neighbors will discard the packet. The channel access protocol is slotted ALOHA which is often used in subscriber-based satellite communication networks and contactless Radio-frequency Identification (RFID) technologies [6]. We assume that all nodes always have packets to send. Time is divided into slots. In every time slot, each node tries to transmit according to a Bernoulli process with parameter $p$, where $0<p \leq 1$. A separate channel is available for acknowledgement. Furthermore, whenever there is a packet waiting to be sent, it is equally likely that this packet will be destined to any node. Relevant terminologies are defined as follows. 


\section{A. Antenna Model and Maximum Transmission Radius $R$}

A smart antenna consists of a main lobe and several parasitic side lobes which can become harmful interference to other receivers in the vicinity. However, the side lobes can be steered toward areas without nodes owing to the adaptability of the directional antenna beams [5]. For simplicity, side lobes are neglected in the rest of the paper, and the beam pattern is modeled as a sector.

By using smart antennas, the transmission radius is extended by shaping the transmission range into a sector. Given the same amount of power, smart antennas can transmit farther than the omni-directional ones. Let $R$ and $R_{\text {omni }}$ be the maximum transmission radii of a smart antenna and an omni-directional antenna, respectively. We assume that the transmission ranges covered by smart and omni-directional antennas are approximately the same. Therefore,

$$
R \approx \sqrt{\frac{2 \pi\left(R_{\text {omni }}\right)^{2}}{\beta}}
$$

where $\beta$ is the beam-width of a smart antenna such that $\beta \in(0,2 \pi)$.

\section{B. Transmission Direction and Range}

Node $A$ with a smart antenna can transmit in a range shaped as a sector with an angle $\beta$ as shown in Fig. 1. This is called the transmission range of $A$. The centerline of the sector is defined as the transmission direction of node $A$. The transmission radius $R_{x}$ falls between 0 and $R$.

\section{Interference Range to Transmission Range Ratio}

As exhibited in Fig. 1, when node $A$ is transmitting with an angle of $\beta$ and a radius of $R_{x}$, the interference range of $A$ is a sector with an angle of $\alpha$ and a radius of $R_{I}$. The transmission direction of $A$ is also the centerline of its interference range. Any node in the transmission range of $A$ can receive $A$ 's signal. However, a node in the interference range but outside the transmission range of $A$, as shown in the shaded area in Fig. 1, cannot receive $A$ 's signal successfully but it will be interfered by the signal.

We set two parameters, namely, $a_{R}$ and $a_{\beta}$, to define the interference range with respect to the transmission range of a node. Specifically, $a_{R}=R_{I} / R_{x}$ and $a_{\beta}=\alpha / \beta$. Here,

$$
\alpha=\left\{\begin{array}{cc}
a_{\beta} \beta, & a_{\beta} \beta \in(0,2 \pi] \\
2 \pi, & a_{\beta} \beta \in(2 \pi, \infty)
\end{array}\right.
$$

If $R_{x}=R, R_{I}$ equals $a_{R} R$, which is the maximum interference radius of a node. We define the circle centered at $A$ with a radius of $a_{R} R$ as the maximum interference range of $A$. Any nodes within this range may be potentially interfered by the transmission of $A$. Furthermore, we define these nodes as the neighbors of $A$.

\section{Forward Progress}

The forward progress of a transmitter $A$ to a receiver $B$ is defined as the distance between $A$ and $B$ projected onto a line drawn from $A$ towards its final destination $C$ [3]. As exhibited in Fig. 2, the forward progress of $A$ to $B$ is $Z$.

As shown in Fig. 2, line $A C$ is defined as the forward direction of $A$, where $C$ is the final destination of node $A$. The middle line is perpendicular to the line $A C$, and cuts the maximum interference range of $A$ into two halves. The shaded semicircle is called the forward semicircle of $A$, and the other one is called the backward semicircle of $A$. Any receiver in the intersection of the forward semicircle and maximum transmission range of $A$ can provide nonnegative forward progress to $A$.

Node $A$ will transmit only if it can find an eligible receiver in its forward semicircle as shown in Fig. 2, since any receiver in $A$ 's backward semicircle cannot contribute a positive forward progress.

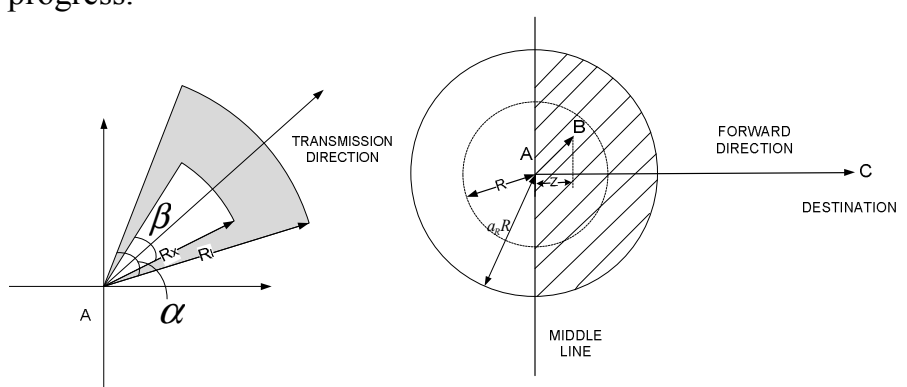

Fig. 1. Transmission range and interference range.

Fig. 2. Forward progress, semicircle, and middle line.

\section{E. Node Distribution}

All nodes in the network are distributed as a two-dimensional Poisson point process with density $\lambda$ (nodes per unit area).

- Define $N$, the mean number of nodes within an area of $\pi R^{2}$, as the network connectivity. Thus, $\lambda$ equals $N / \pi R^{2}$.

- $\quad$ The probability of finding $i$ nodes in an area of size $G$ is $(\lambda G)^{i} e^{-\lambda G} / i$ !, where $i=1,2,3, \ldots$.

- Let $A+$ be the event that node $A$ can find an eligible receiver in the forward semicircle. By [3], $P_{r}(A+)=1-\left(\lambda \pi R^{2} / 2\right)^{0} e^{-\lambda \frac{1}{2} \pi R^{2}} / 0 !=1-e^{-N / 2}$.

\section{PROBABILITY OF INTERFERENCE}

\section{A. Analysis of Interference}

Suppose node $A$ wants to send a packet to its final destination $C$ and $B$ is the first relay of this packet. Thus, a transmission occurs from node $A$ to node $B$. For this transmission to be successful, $B$ cannot be covered by the interference ranges of its neighbors (excluding node $A$ ).

Let $M$ be one of $B$ 's neighbors other than $A$ and $M$ also has a packet to be sent. By the network model described in Section II, neighbors of a node are uniformly distributed in the maximum interference range of that node. Thus, $B$ is uniformly distributed within the maximum interference range of $M$. Define a polar coordinate system with respect to $M$. Denote the polar coordinate of $B$ as $B\left(r_{M B}, \phi_{M B}\right)$, where $r_{M B}$ is the distance between $M$ and $B$, and $\phi_{M B}$ is the polar angle measured from the forward direction of $M$ to the direction to reach $B$. 


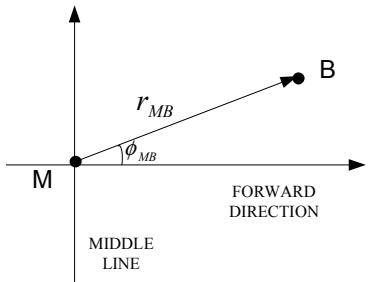

Fig. 3. An illustration for the polar coordinate of $M$.

By [3], the probability density functions of $r_{M B}$ and $\phi_{M B}$ are computed as:

$$
\begin{gathered}
f_{r_{M B}}\left(r_{m b}\right)=2 \pi r_{m b} / \pi\left(a_{R} R\right)^{2}=2 r_{m b} /\left(a_{R} R\right)^{2}, \quad r_{m b} \in\left(0, a_{R} R\right] \\
f_{\left.\phi_{M B}\right|_{M B}}\left(\phi_{m b} \mid r_{m b}\right)=1 / 2 \pi, \quad \phi_{m b} \in[0,2 \pi)
\end{gathered}
$$

Thus $f_{r_{M B}, \phi_{M B}}\left(r_{m b}, \phi_{m b}\right)=f_{r_{M B}}\left(r_{m b}\right) \cdot f_{\phi_{M B} \mid r_{M B}}\left(\phi_{m b} \mid r_{m b}\right)=r_{m b} / \pi\left(a_{R} R\right)^{2}$

\section{B. Probability of Interference for NFP}

In NFP, the transmitter chooses the nearest node that can provide positive forward progress. The transmission radius is just the distance between the transmitter and the receiver.

Let $I$ be the event that $B$ gets interfered by $M$. The maximum interference range of $M$ is divided into four divisions as shown in Fig. 4. The probability of $I$ is computed based on these four divisions. That is, the probability of $I$ is the sum of four probabilities, corresponding to the case that $B$ is in one of the four divisions and gets interfered. Let $\operatorname{Div}(i)$ be the event that $B$ is in division $i$, where $i=1,2,3,4$. According to the Law of Total Probability, we can get:

$$
P_{r}(I)=\sum_{i=1}^{4} P_{r}(I, \operatorname{Div}(i))
$$

\section{1) Case 1: Computation of $P_{r}(I, \operatorname{Div}(1))$}

In this case, $P_{r}(I, D i v(1))$ is the probability that $B$ is in division 1 and gets interfered by $M$.

Let $\operatorname{Rev} M$ be the receiver of $M$. Here, RevM is the first relay of the packet sent by $M . r_{M}$ is the transmission radius of $M$. By [3], the probability density function of a receiver's position for a transmission using NFP can be computed as:

$$
f_{r, \theta}^{N F P}\left(r_{0}, \theta_{0}\right)=\frac{\lambda r_{0} e^{-\lambda \pi_{0}^{2} / 2}}{1-e^{-N / 2}} \quad, r_{0} \in(0, R], \theta_{0} \in[-\pi / 2, \pi / 2]
$$

where $r$ and $\theta$ are the distance between $M$ and $\operatorname{Rev} M$, and transmission angle of $M$, respectively. The transmission angle of a node is defined as the polar angle between the transmission direction and forward direction. Note that the transmission radius $r_{M}$ is equal to $r$ in NFP, i.e. $r_{M}=r$.

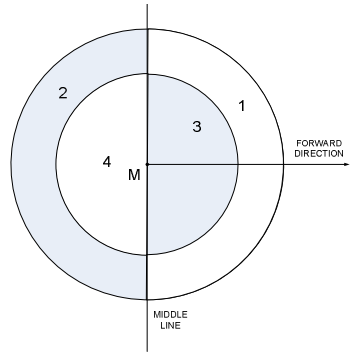

Fig. 4. An illustration for the four divisions. The probability of the event $\{I, \operatorname{Div}(1)\}$ is equivalent to the probability of the event $\left\{M+, B \in \Psi_{M \rightarrow \operatorname{Re} v M}, \operatorname{Div}(1)\right\}$, where $M+$ is the event that $M$ can find an eligible receiver, and $B \in \Psi_{M \rightarrow \operatorname{Re} v M}$ is the event that node $B$ is in the interference range of the transmission from $M$ to $\operatorname{Rev} M$. Now, $P_{r}(M+)=1-e^{-\frac{N}{2}}$. The event $\left\{B \in \Psi_{M \rightarrow \operatorname{Re} v M}, D(1) \mid M+\right\}$ occurs when the following three conditions are all satisfied:

$$
\begin{aligned}
& \text { 1. } r_{M B} \in\left(R, a_{R} R\right] \text { and } \phi_{M B} \in[-\pi / 2, \pi / 2] ; \\
& \text { 2. } r<r_{M B} \leq a_{R} \cdot r_{M} ; \text { and } \\
& \text { 3. }\left|\theta-\phi_{M B}\right| \leq \alpha / 2 .
\end{aligned}
$$

The first condition is for node $B$ to be in the division 1 . The second and the third conditions specify $B$ is in the interference range of the transmission from $M$ to $\operatorname{Rev} M, \Psi_{M \rightarrow \operatorname{Rev} M}$.

Since $r$ is the distance between $M$ and $\operatorname{Rev} M, r \in(0, R]$. As

$$
\begin{aligned}
& r_{M}=r \text { and } r<r_{M B} \leq a_{R} r_{M}, \text { we can get } r_{M B} / a_{R} \leq r<R . \\
& P_{r}(I, D i v(1))=P_{r}\left(M+, B \in \Psi_{M \rightarrow \operatorname{Re} v M}, \operatorname{Div}(1)\right) \\
&= P_{r}(M+) \cdot P_{r}\left(B \in \Psi_{M \rightarrow \operatorname{Re} v M}, \operatorname{Div}(1) \mid M+\right) \\
&= P_{r}(M+) \cdot P_{r}\left(B \in \Psi_{M \rightarrow \operatorname{Rev} M} \mid \operatorname{Div}(1), M+\right) \cdot P_{r}(\operatorname{Div}(1) \mid M+) \\
&= P_{r}(M+) \cdot \int_{R}^{a_{R} R} \int_{-\pi / 2}^{\pi / 2} f_{r_{M B}, \phi_{M B}}\left(r_{m b}, \phi_{m b}\right) \cdot P_{r}\left(B \in \Psi_{M \rightarrow \operatorname{Rev} M} \mid \operatorname{Div}(1), M+\right) d \phi_{m b} d r_{m b} \\
&=\left(1-e^{-N / 2}\right) \int_{R}^{a_{R} R} \int_{-\pi / 2}^{\pi / 2} \frac{r_{m b}}{\pi\left(a_{R} R\right)^{2}} \cdot \int_{r_{m b} / a_{R}}^{R} \int_{\phi_{1}}^{\phi_{2}} f_{r, \theta}^{N F P}\left(r_{0}, \theta_{0}\right) d \theta_{0} d r_{0} d \phi_{m b} d r_{m b} \\
&= \frac{\lambda}{\pi\left(a_{R} R\right)^{2}} \int_{R}^{a_{R} R} \int_{-\pi / 2}^{\pi / 2} \int_{r_{m b} / a_{R}}^{R} \int_{\phi_{1}}^{\phi_{2}} r_{m b} r_{0} e^{-\frac{\lambda m_{0}^{2}}{2}} d \theta_{0} d r_{0} d \phi_{m b} d r_{m b}
\end{aligned}
$$

Where

$$
\phi_{1}=\max \left\{\left(\phi_{m b}-\alpha / 2\right),-\pi / 2\right\}
$$

and

$\phi_{2}=\min \left\{\left(\phi_{m b}+\alpha / 2\right), \pi / 2\right\}$.

Since $M$ will only transmit when it finds a receiving node in its forward semicircle, there are boundary effects when $B$ is located close to the middle line of $M$. For example, when $\phi_{M B}=\pi / 2$, the range of integration on $\theta$ should be $[\pi / 2-\alpha / 2, \pi / 2]$. Since the probability density function $f_{r, \theta}^{N F P}\left(r_{0}, \theta_{0}\right)$ will be non-zero only for $\theta$ in the range of $[-\pi / 2, \pi / 2]$, we have $\phi_{1}=\max \left\{\left(\phi_{m b}-\alpha / 2\right),-\pi / 2\right\}$ and $\phi_{2}=\min \left\{\left(\phi_{m b}+\alpha / 2\right), \pi / 2\right\}$.

2) Case 2: Computation of $P_{r}(I, \operatorname{Div}(2))$

In this case, $P_{r}(I, \operatorname{Div}(2))$ is the probability that $B$ is in division 2 and gets interfered by $M$.

$B$ will be interfered only if the interference range of $M$ is extended to the backward semicircle as exhibited in Fig. 6. We can see that when the transmission direction of $M$ is within either of the dashed areas, each of which is a sector with an angle of $\alpha / 2$, its interference range will spread to the backward semicircle.

The event $\left\{B \in \Psi_{M \rightarrow \operatorname{Re} v M}, D(2) \mid M+\right\} \quad$ occurs when the following three conditions are all satisfied:

$$
\begin{array}{ll}
\text { 1. } & r_{M B} \in\left(R, a_{R} R\right] \text { and } \phi_{M B} \in\left[\frac{\pi}{2}, \frac{\pi}{2}+\frac{\alpha}{2}\right] \cup_{\phi_{M B} \in\left(-\frac{\pi}{2}-\frac{\alpha}{2},-\frac{\pi}{2}\right]} ; \\
\text { 2. } & 0<r_{M B} \leq a_{R} \cdot r_{M} \text {; and } \\
\text { 3. } & \left|\theta-\phi_{M B}\right| \leq \alpha / 2 .
\end{array}
$$

Since $r \in(0, R], r_{M}=r$, and $0<r_{M B} \leq a_{R} r_{M}$, we can get 
$r_{M B} / a_{R} \leq r<R$.

There are two different cases on $\alpha$ ( $\alpha$ is the interference angle), namely, $\alpha \in(0, \pi]$ and $\alpha \in(\pi, 2 \pi]$. Consider when $\alpha \in(0, \pi]$. For $\phi_{M B} \in(\pi / 2, \pi / 2+\alpha / 2]$, an edge of the interference range of $M$ being within $\left[\phi_{M B}, \pi / 2+\alpha / 2\right]$ will make the interference range of $M$ cover $M B$ as shown in Fig. 6(a), where the interference range of $M$ is the shaded sector. It corresponds to the case that $M$ 's transmission direction is within the range $\left[\phi_{M B}-\alpha / 2, \pi / 2\right]$ as shown in the dark grey sector in Fig. 6(a). For $\phi_{M B} \in(-\pi / 2-\alpha / 2,-\pi / 2]$, an edge of the interference range of $M$ being within $\left[-\pi / 2-\alpha / 2, \phi_{M B}\right]$ will make the interference range of $M$ cover $M B$ as shown in Fig. 6(b). It corresponds to the case that M's transmission direction is within the range $\left[-\pi / 2, \phi_{M B}+\alpha / 2\right]$ as shown in the dark grey sector in Fig. 6(b).

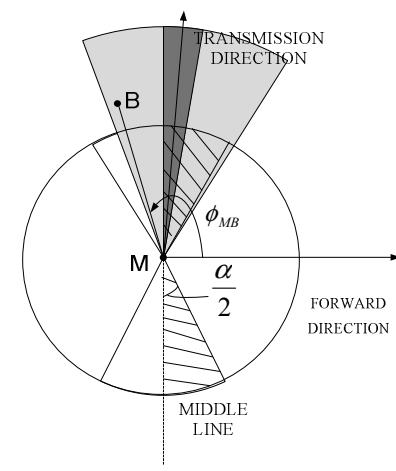

(a) $\phi_{M B} \in(\pi / 2, \pi / 2+\alpha / 2]$.

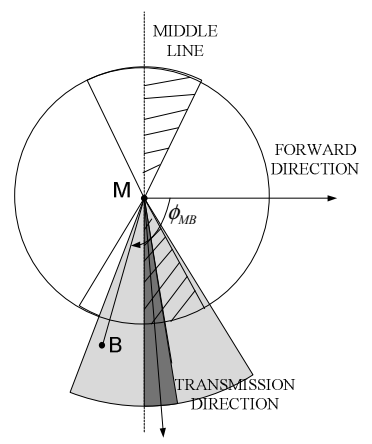

(b) $\phi_{M B} \in(-\pi / 2-\alpha / 2,-\pi / 2]$.

Fig. 6. An illustration for $B$ in the backward semicircle of $M$.

Thus, when $\alpha \in(0, \pi]$,

$$
\begin{aligned}
& P_{r}(I, \operatorname{Div}(2))=P_{r}\left(M+, B \in \Psi_{M \rightarrow \operatorname{Re} v M}, \operatorname{Div}(2)\right) \\
= & P_{r}(M+) \cdot P_{r}\left(B \in \Psi_{M \rightarrow \operatorname{Re} v M}, \operatorname{Div}(2) \mid \operatorname{Div}(2), M+\right) \cdot P_{r}(\operatorname{Div}(2) \mid M+) \\
= & P_{r}(M+) \cdot \int_{R}^{a_{R} R} \int_{\pi / 2}^{\pi / 2+\alpha / 2} f_{r_{M B}, \phi_{M B}}\left(r_{m b}, \phi_{m b}\right) \cdot P_{r}\left(B \in \Psi_{M \rightarrow \operatorname{Re} v M} \mid \operatorname{Div}(2), M+\right) d \phi_{m b} d r_{m b} \\
& +\int_{R}^{a_{R} R} \int_{-\pi / 2-\alpha / 2}^{-\pi / 2} f_{r_{M B}, \phi_{M B}}\left(r_{m b}, \phi_{m b}\right) \cdot P_{r}\left(B \in \Psi_{M \rightarrow \operatorname{Re} v M} \mid \operatorname{Div}(2), M+\right) d \phi_{m b} d r_{m b} \\
= & \frac{\lambda^{2}}{\pi^{2}\left(a_{R} R\right)^{4}} \cdot\left(\int_{R}^{a_{R} R} \int_{\pi / 2}^{\pi / 2+\alpha / 2} \int_{\phi_{m b}-\alpha / 2}^{\pi / 2} \int_{r_{m b} / a_{R}}^{R} r_{m b} r_{0}^{2} e^{-\frac{\lambda m_{0}^{2}}{2}} d r_{0} d \theta_{0} d \phi_{m b} d r_{m b}\right. \\
& \left.\int_{R}^{a_{R} R} \int_{-\pi / 2-\alpha / 2}^{-\pi / 2} \int_{-\pi / 2}^{\alpha / 2+\phi_{m b}} \int_{r_{m b} / a_{R}}^{R} r_{m b} r_{0}^{2} e^{-\frac{\lambda m_{0}^{2}}{2}} d r_{0} d \theta_{0} d \phi_{m b} d r_{m b}\right)
\end{aligned}
$$

When $\alpha \in(\pi, 2 \pi]$, the edge of the interference range of $M$ being within either $\left[\phi_{M B}, \pi / 2+\alpha / 2\right]$ or $\left[-\pi / 2-\alpha / 2, \phi_{M B}\right]$ can possibly make the interference range of $M$ cover $M B$. It corresponds to the case that $M$ 's transmission direction is within the range $\left[\phi_{M B}-\alpha / 2, \pi / 2\right] \cup\left[-\pi / 2, \phi_{M B}+\alpha / 2\right]$.

Hence, when $\phi_{M B} \in[\pi / 2, \pi / 2+\alpha / 2] \cup(-\pi / 2-\alpha / 2,-\pi / 2]$, and $r_{M B} \in\left(R, a_{R} R\right]$, using an approach as in (2), we can get:

$$
\begin{aligned}
& P_{r}\left(B \in \Psi_{M \rightarrow \operatorname{Re} v M} \mid \operatorname{Div}(2), M+\right) \\
& =\int_{\phi_{m b}-\frac{\alpha}{2}}^{\frac{\pi}{2}} \frac{\int_{\frac{r_{M B}}{a_{R}}}^{R}}{r_{r, \theta}} f^{N F P}\left(r_{0}, \theta_{0}\right) d r_{0} d \theta_{0}+\int_{-\pi / 2}^{\alpha / 2+\phi_{m b}} \int_{r_{m b} / a_{R}}^{R} f_{r, \theta}^{N F P}\left(r_{0}, \theta_{0}\right) d r_{0} d \theta_{0}
\end{aligned}
$$

Thus, $P_{r}\left(B \in \Psi_{M \rightarrow \operatorname{Rev} M}, \operatorname{Div}(2) \mid M+\right)$ is equal to the integration of $f_{r_{M B}, \phi_{M B}}\left(r_{m b}, \phi_{m b}\right) \cdot P_{r}\left(B \in \Psi_{M \rightarrow \operatorname{Rev} M} \mid \operatorname{Div}(2)\right) \quad$ on the range $\phi_{m b} \in[\pi / 2, \pi / 2+\alpha / 2] \cup(-\pi / 2-\alpha / 2,-\pi / 2]$ and $r_{m b} \in\left(R, a_{R} R\right]$.
3) Case 3: Computation of $P_{r}(I, \operatorname{Div}(3))$

In this case, $P_{r}(I, \operatorname{Div}(3))$ is the probability that $B$ is in division 3 and gets interfered by $M$.

Since $B$ is within the intersection of maximum transmission range and forward semicircle of $M, M$ can always find a receiver to send a packet to. The probability of the event $\{I, \operatorname{Div}(3)\}$ is equivalent to the probability of the event $\left\{B \in \Psi_{M \rightarrow \operatorname{Re} v M}, D(3)\right\}$.

In Fig. 7, the largest sector is the interference range of $M$. Since $\operatorname{Rev} M$ is the closest node to $M$ in NFP, $r$ must be less than $r_{M B}$.

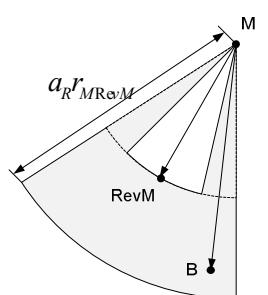

Fig. 7. An illustration for $B$ to be interfered when $r_{M B}<R$.

Since $\operatorname{Rev} M$ is the closest node to $M$ in NFP, $r$ must be less than $r_{M B}$. The event $\left\{B \in \Psi_{M \rightarrow \operatorname{Rev} M}, D(3)\right\}$ occurs when the following three conditions are all satisfied:

$$
\begin{aligned}
& \text { 1. } r_{M B} \in(0, R] \text { and } \phi_{M B} \in[-\pi / 2, \pi / 2] ; \\
& \text { 2. } \quad r<r_{M B} \leq a_{R} \cdot r_{M} ; \text { and } \\
& \text { 3. }\left|\theta-\phi_{M B}\right| \leq \alpha / 2 . \\
& \text { Since } r \in(0, R], r_{M}=r \text {, and } r<r_{M B} \leq a_{R} r_{M} \text {, we can get } \\
& r_{M B} / a_{R} \leq r<r_{M B} . \\
& P_{r}(I, \operatorname{Div}(3))=P_{r}\left(B \in \Psi_{M \rightarrow \operatorname{Rev} M}, \operatorname{Div}(3)\right) \\
& =P_{r}\left(B \in \Psi_{M \rightarrow \operatorname{Re} v M} \mid \operatorname{Div}(3)\right) \cdot P_{r}(\operatorname{Div}(3)) \\
& =\int_{0-\pi / 2}^{R} \int_{r_{M B}, \phi_{M B}}\left(r_{m b}, \phi_{m b}\right) \cdot P_{r}\left(B \in \Psi_{M \rightarrow \operatorname{Re} v M} \mid \operatorname{Div}(3)\right) d \phi_{m b} d r_{m b} \\
& =\frac{\lambda}{\pi\left(a_{R} R\right)^{2}\left(1-e^{-\frac{N}{2}} \int_{0}^{R} \int_{-\pi / 2}^{\pi / 2} \int_{r_{m b} / a_{R}}^{r_{m b}} \int_{\phi_{1}}^{\phi_{2}} r_{m b} \cdot r_{0} \cdot e^{-\frac{\lambda m_{0}^{2}}{2}} d \theta_{0} d r_{0} d \phi_{m b} d r_{m b}\right.}
\end{aligned}
$$

where $\phi_{1}=\max \left\{\left(\phi_{m b}-\frac{\alpha}{2}\right),-\frac{\pi}{2}\right\}$ and $\phi_{2}=\min \left\{\left(\phi_{m b}+\frac{\alpha}{2}\right), \frac{\pi}{2}\right\}$. Note that the boundary effect of $\theta$ is the same as that in Case 1 .

4) Case 4: Computation of $P_{r}(I, \operatorname{Div}(4))$

In this case, $P_{r}(I, D i v(4))$ is the probability that $B$ is in division 4 and gets interfered by $M$.

$$
P_{r}(I, \operatorname{Div}(4))=P_{r}\left(M+, B \in \Psi_{M \rightarrow \operatorname{Re} v M}, \operatorname{Div}(4)\right)
$$

The event $\left\{B \in \Psi_{M \rightarrow \operatorname{Rev} M}, \operatorname{Div}(4) \mid M+\right\}$ occurs when the following three conditions are all satisfied:

$$
\begin{aligned}
& \text { 1. } r_{M B} \in(0, R], \phi_{M B} \in[\pi / 2, \pi / 2+\alpha / 2] \cup \phi_{M B} \in(-\pi / 2-\alpha / 2,-\pi / 2] ; \\
& \text { 2. } 0<r_{M B} \leq a_{R} \cdot r_{M} ; \text { and } \\
& \text { 3. }\left|\theta-\phi_{M B}\right| \leq \alpha / 2 .
\end{aligned}
$$

The argument is similar to that for Case 2. The only difference is that $B$ is within the maximum transmission range of $M$ for Case 4 . We get similar equations as equations (2), (3) in Case 2, and the only modifications are the upper and lower bounds of $r_{M B}$ are changed to $R$ and 0 , respectively.

In Fig. 8, we plot $P_{r}(I)$ versus connectivity $N$ with different 
values of $\beta$. By using smart antennas, $P_{r}(I)$ is reduced as $\beta$ shrinks. The reason is as follows. In NFP, a transmitter chooses the nearest node in its forward semicircle as its receiver. The transmission radius is the distance between the transmitter and the receiver. As $\beta$ shrinks, the interference range becomes smaller. Therefore, a node is less likely to be interfered. The probability of the interference is the largest when the beam-width $\beta$ equals to $2 \pi$ which corresponds to the case of the omni-directional antennas.

Moreover, with adjustable transmission power, $P_{r}(I)$ remains comparably stable with increasing $N$. As the node density of the network increases, a transmitter is more likely to choose a closer node as its receiver. Therefore, the transmission radius is reduced. Even if there is more traffic in the network with increasing $N$, the interference will not increase dramatically since the transmission ranges, and hence the interference ranges, of the transmitters become smaller.

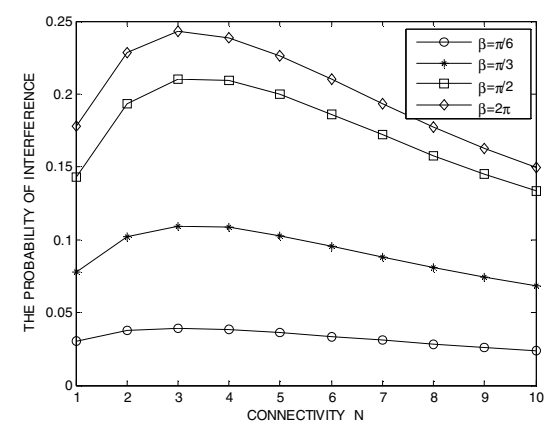

Fig. 8 . The probability of interference versus connectivity $N$ with various values of $\beta$ under NFP.

\section{PERFORMANCE ANALYSIS}

We assume that all nodes in the network transmit with probability $p . I^{c}$ is the event that $M$ does not interfere with $B . M^{t}$ is the event that $M$ transmits, while $M^{c}$ is the event that it does not transmit.

$$
\begin{aligned}
P\left(I^{c}\right) & =P\left(I^{c} \mid M^{c}\right) \cdot P\left(M^{c}\right)+P\left(I^{c} \mid M^{t}\right) \cdot P\left(M^{t}\right) \\
& =1 \cdot(1-p)+\left(1-P_{r}(I)\right) \cdot p
\end{aligned}
$$

Let $N_{i}$ be the event that $B$ has $i$ neighbors excluding node $A$, and $T_{A B}$ be the event that when there is a transmission from $A$ to $B$, these $i$ neighbors will not interfere with $B$.

$$
P_{r}\left(T_{A B} \mid N_{i}\right)=(1-p) \prod_{M=1}^{i} P_{r}\left(I^{c}\right)=(1-p)\left(1-p \cdot P_{r}(I)\right)^{i}
$$

We assume that $a_{R}=a_{\beta}=3 / 2$ for our study.

Since $B$ is the nearest node to $A$ that can provide positive forward progress, there is no node in the semicircle between $A$ and $B$ as illustrated in Fig. 9. We call this area the "excluded region" and denote it as $E$. Here, $E=1 / 2 \cdot \pi r^{2}$.

The excluded region $E$ is included in the maximum interference range of $B$, which is a circle with radius $a_{R} R$. The number of $B$ 's neighbors (excluding $A$ ) can be calculated as:

$P_{r}\left(N_{i} \mid B(r, \theta)\right)=\frac{\left(\lambda\left(\pi\left(a_{R} R\right)^{2}-E\right)\right)^{i}}{i !} \cdot e^{-\lambda\left(\pi\left(a_{R} R\right)^{2}-E\right)}$ where $\mathrm{i}=0,1,2, \ldots$

Since $B$ is the receiver of $A, r \in(0, R]$, and $\theta \in[-\pi / 2, \pi / 2]$.

$$
\begin{aligned}
& P_{r}\left(T_{A B}\right)=\sum_{i=0}^{\infty} P_{r}\left(T_{A B} \mid N_{i}\right) \cdot P_{r}\left(N_{i}\right) \\
= & \sum_{i=0}^{\infty} P_{r}\left(T_{A B} \mid N_{i}\right) \cdot \int_{0}^{R} \int_{-\pi / 2}^{\pi / 2} P_{r}\left(N_{i} \mid B=\left(r_{0}, \theta_{0}\right)\right) \cdot f_{r, \theta}\left(r_{0}, \theta_{0}\right) d r_{0} d \theta_{0} \\
= & \int_{0}^{R} \int_{-\pi / 2}^{\pi / 2}(1-p) \cdot \exp \left(-p \cdot P_{r}(I) \lambda\left(\pi\left(a_{R} R\right)^{2}-E\right)\right) \cdot f_{r, \theta}\left(r_{0}, \theta_{0}\right) d r_{0} d \theta_{0}
\end{aligned}
$$

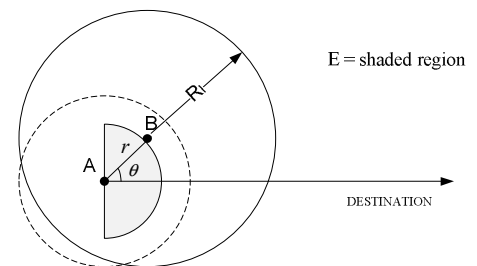

Fig. 9. An illustration of the excluded region for NFP.

We use the following metrics to evaluate the network performance.

$S=$ one-hop throughput

$=$ average number of successful transmissions per slot from a node

$Z=$ average forward progress per slot from a node

Let $A+$ be the event that $A$ can find a receiver for the transmission, and $A^{t}$ the event that $A$ transmits. Therefore, the local throughput at node $A$ can be computed as:

$$
S=P_{r}(A+) \cdot P_{r}\left(A^{t}\right) \cdot P_{r}\left(T_{A B}\right)=\left(1-e^{-N / 2}\right) \cdot p \cdot P_{r}\left(T_{A B}\right)
$$

The forward progress is equal to $r \cos \theta$, where $r$ and $\theta$ are the transmission radius and angle of $A$, respectively. We can get the average forward progress $Z$ by inserting $r \cos \theta$ into the integral above:

$$
\begin{aligned}
Z= & \left(1-e^{-N / 2}\right) \cdot p \cdot(1-p) . \\
& \int_{0}^{R} \int_{-\pi / 2}^{\pi / 2} \exp \left(-p P_{r}(I) \lambda\left(\pi\left(a_{R} R\right)^{2}-E\right)\right) \cdot r_{0} \cos \theta_{0} \cdot f_{r, \theta}\left(r_{0}, \theta_{0}\right) d \theta_{0} d r_{0}
\end{aligned}
$$

We write a computer program in Visual $\mathrm{C}++$ to simulate an area of $15 R \times 15 R$ to validate our analytical model, where $\mathrm{R}$ is the logical maximum transmission range. The nodes are distributed according to a two-dimensional Poisson point process. The position of each node is denoted as $(\mathrm{x}, \mathrm{y})$, where $\mathrm{x}$ and $\mathrm{y}$ are uniformly distributed in $[0,15 R]$. The network connectivity $N$ varies from one to ten and the number of nodes in the network is $15 R \times 15 R \times N /\left(\pi R^{2}\right)=225 N / \pi$.

1. First, we identify the typical nodes which are located in the $13 R \times 13 R$ square centered at the middle of the simulated area so as to omit the edge effect. Denote by $F$ the number of typical nodes.

2. For each transmission-destination pair, the next relay for the transmitter is determined according to NFP.

3. Each node in the network transmits with probability $p$. For each transmission from a transmitter to its next relay, we check every typical node to see whether it is covered by the interference range of this transmission.

4. We identify the typical nodes that are not covered by any interference ranges of others. If the typical node is a relay, the transmission to it is said to be successful and the forward progress of this transmission is recorded. Denote $H$ as the number of typical nodes with successful transmissions. 
5. The one-hop throughput $S$ is computed as $H / F$.

6. Let $Z_{\text {sum }}$ be the sum of the recorded forward progresses. The average forward progress per slot from a node $Z$ is computed as $Z_{\text {sum }} / H$.

For each value of $N, p$ is chosen to maximize $Z \sqrt{\lambda}$. The analytical and simulation results for one-hop throughput and the normalized forward progress are shown in Figs. 10 and 11. Each data point obtained from simulation is an average value of 100 simulation runs. The confidence intervals have not been drawn since they are too small to be shown in the figure. In general, the analytical and simulation results follow similar trends, but there are still some discrepancies. The approximation and independence assumptions we use in the analysis may be the reasons.

As we can see from Fig. 10 and 11, the curves of $S$ and $Z \sqrt{\lambda}$ rise at first, and have a stable trend as $N$ grows. This is because when the network connectivity is low, it is difficult for a node to find a receiver. The network performance will be improved when the network becomes more connected. However, there is always a tradeoff between performance and network connectivity. As $N$ grows larger, more network traffic will lead to more interference, thus deteriorating the network performance. Therefore, $S$ and $Z \sqrt{\lambda}$ do not increase any more.

Moreover, with adjustable transmission power, both the one-hop throughput $S$ and normalized average progress $Z \sqrt{\lambda}$ remain stable with respect to varying network connectivity. As the network connectivity increases, a transmitter is more likely to find a closer node as its receiver. Therefore, the transmission radius is reduced. Even if there is more traffic in the network and $N$ increases, the interference will not increase dramatically, since the transmission ranges of the transmitters become smaller.

\section{CONCLUSION}

We present a model to analyze the throughput and forward progress in wireless ad hoc networks with power-adjustable directional antennas. Our model can accommodate different transmission and interference ranges. It uses two parameters, namely $a_{R}$ and $a_{\beta}$, to define the interference ratio. Our results show that performance can be greatly improved by using directional antennas instead of omni-directional antennas. Adjustable transmission radius can also mitigate the interference in the network and ensure performance stability. Furthermore, NFP always yields stable throughput and average forward progress with respect to varying node densities. However, for the multi-hop applications in which forward progress is a key measure of performance, NFP may not have the best performance. In the future, we will study other transmission strategies.

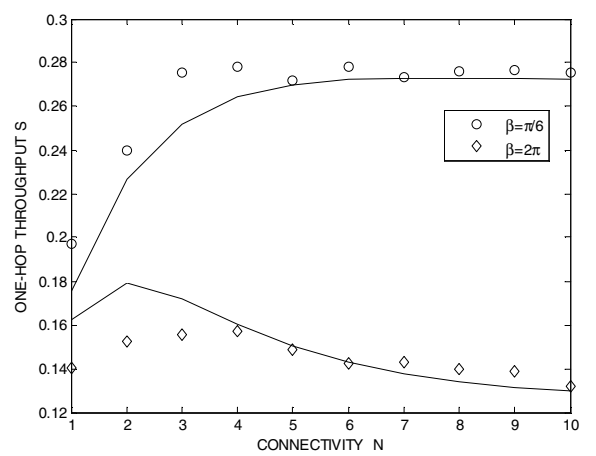

Fig. 10. One-hop throughput $S$ versus connectivity $N$.

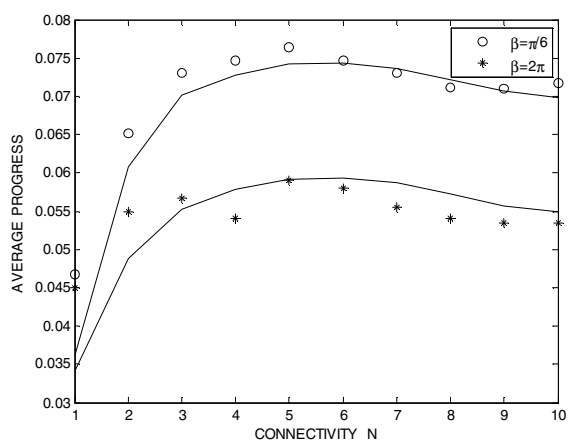

Fig. 11. Average forward progress versus connectivity $N$.

\section{REFERENCES}

[1] R. Compton, Adaptive Antennas: Concepts and Performance. Prentice Hall, 1988.

[2] J. Deng, Y. S. Han, P. N. Chen and P. K. Varshney, "Optimal transmission range for wireless ad hoc networks based on energy efficiency," IEEE Trans. Communications, Vol. 55, No. 9, Sep. 2007.

[3] T. C. Hou and V. O. K. Li, "Transmission range control in mulitihop packet radio networks," IEEE Trans. Communications, Vol. COM-34, No.1, Jan. 1986.

[4] E. D. Kaplan, Ed., Understanding GPS: Principles and Applications. Boston, MA: Artech House, 1996.

[5] C. B. Li, J. J. Garcia-Luna-Aceves, "Transmission scheduling in ad hoc networks with directional antennas," in Proc. MOBICOM'02, pp. 48-58, Sep. 2002.

[6] Q. Liu and J. Li, "Packet delivery through difficult wireless channel," in Proc. MILCOM, Vol. 1, pp. 530-535, Oct. 2002.

[7] H.-J. Perz and B. Walke, "Adjustable transmission power for mobile communications with ominidirectional and directional antennas in an one-and-multi-hop environment," in Proc. IEEE Vehicular Technology Conference, pp. 630-635, May. 1991.

[8] J. F. Shan, C. Zheng, H. Wang and N. H. Yu, "An effective MAC scheme based on large interference range in mobile ad hoc networks," in Proc. 6th International Conference on Machine Learning and Cybernetics, Vol. 4, pp. 2145-2149, Aug, 2007.

[9] S. L. Wu, Y. C. Tseng and J. P. Sheu, "Intelligent medium access for mobile ad hoc networks with busy tones and power control," IEEE Trans. Communications, Vol. 18, No. 9, Sep. 2000.

[10] J. Zander, "Slotted aloha multihop packet radio networks with directional antennas," Electronic Letters, Vol.26, No.25, pp. 2098-2100, Dec. 1990. 